\title{
Methods for Extraction, Isolation and Purification of C-phycocyanin: 50 years of Research in Review
}

\author{
Verónica Cruz de Jesús ${ }^{1}$, Gabriel Alfonso Gutiérrez-Rebolledo², Marcela Hernández-Ortega ${ }^{3}$, Lourdes \\ Valadez-Carmona4, Angélica Mojica-Villegas ${ }^{2}$, Gabriela Gutiérrez-Salmeán ${ }^{5 \S}$ and Germán Chamor- \\ ro-Cevallos ${ }^{2 *}$
}

${ }^{1}$ Faculty of Higher Cuautitlán Izcalli, National Autonomous University of Mexico, Mexico state, MéxicO

${ }^{2}$ Department of Pharmacy, National School of Biological Sciences of the National Polytechnic Institute. Mexico

${ }^{3}$ Faculty of Chemistry, University of the State of Mexico . Mexico

${ }^{4}$ Biochemical Engineering Laboratory, National School of Biological Sciences, National Polytechnic Institute. Mexico

${ }^{5}$ Faculty of Health Sciences. Universidad Anahuac Mexico Norte. Mexico

${ }^{\S}$ Gutiérrez-Salmeán and Chamorro-Cevallos co-senior this manuscript

*Corresponding author: German Chamorro-Cevallos, Department of Pharmacy, National School of Biological Sciences, National Polytechnic Institute, Avenida Wilfrido Massieu s / n. Unit Adolfo Lopez Mateos, Del. Gustavo A. Madero, 07738 Mexico, DF, Mexico, Tel: +(52) (55) 5729 6000/ ext. 52398; E-mail: gchamcev@yahoo.com.mx

\begin{abstract}
Context: Spirulina (Arthrospira) exerts a wide spectrum of pharmacological activities that are largely attributed to its phycobiliprotein content, mainly to C-phycocyanin. The extraction, isolation and purification of C-phycocyanin have been studied for many years, resulting in diverse methodologies with a range of yields and grades of purity.

Objective: We performed a systematic review of the literature, consulting all the available years in TOXNET, PubMed/MEDLINE and Science Direct-Scopus. Search criteria included the separation, isolation, and purification methods for C-phycocyanin from different microorganisms. Search words were: extraction, separation, isolation and purification of C-phycocyanin.

Results: The combination of aqueous two-phase systems for extraction and ultrafiltration for purification results in the best yields and highest purity of the desired nutraceuticals. It is also essential to consider the freshness and species of the primary biomass, as these factors heavily influence the concentration and viability of the phycobiliproteins and therefore affect the yield and purity.

Conclusion: In order to preserve the valuable properties and health benefits of nutraceuticals, such as C-phycocyanin, it is essential to seek innovative methods for isolating and purifying these bioactive substances from natural sources. The information herein gathered indicates the best methods currently available.
\end{abstract} Received Date: May 25, 2016 Accepted Date: June 10, 2016 Published Date: June 15, 2016

Citation: Chamorro-Cevallos, G., et al. Methods for Extraction, Isolation and Purification of C-phycocyanin: 50 years of Research in Review. (2016) Int J Food Nutr Sci 3(1): 275-284.

DOI: $10.15436 / 2377-0619.16 .946$

Keywords: C-phycocyanin; Extraction; Isolation; Phycobiliproteins; Purification; Spirulina

\section{Introduction}

Spirulina spp, or Arthrospira, is a microscopic and filamentous cyanobacteria with a wide variety of applications including its use as a food source; in fact, Spirulina has been used as food in Mexico since pre-Hispanic times. (Dillon, Phuc, \& Dubacq, ${ }^{[1]}$; Venkataraman, ${ }^{[2]}$.

Nutritional and functional properties of Spirulina

The popularity of Spirulina as a food supplement is due to its high protein content (about $70 \%$ of its dry weight) and high 
biological value (containing essential amino acids like phenylalanine and methionine). Spirulina also contains vitamins: $\mathrm{B}_{12}$ (cyanocobalamin), $\mathrm{B}_{6}$ (pyridoxine), $\mathrm{B}_{1}$ (thiamine) and $\mathrm{B}_{2}$ (riboflavin), as well as beta carotenes (precursors of vitamin A); minerals (e.g., iron, zinc, selenium, calcium and magnesium), phytochemicals (phenolic acids and tocopherols), and essential fatty acids (such as gamma linoleic acid) (Belay, ${ }_{,[3]}^{[3}$ Dillon et al., ${ }^{[1]}$; Habib et al., 2008).

Spirulina is considered a functional food because of its broad spectrum of biologic effects, which have been demonstrated in vitro and in vivo. Among these, Spirulina has been reported to exhibit anti-inflammatory (Remirez, Ledón, \& González, $\left.{ }^{[4]}\right)$, anti-hyperlipidemic (Torres-Duran, Ferreira-Hermosillo, \& Juarez-Oropeza, ${ }^{[5]}$ ), hypoglycemic (Lima, Facchinetti, \& Santos, ${ }^{[6]}$ ), antihypertensive (Torres-Duran et al., ${ }^{[5]}$ ), antineoplastic (Mittal, Suresh Kumar, Banerjee, Rao, \& Kumar, ${ }^{[7]}$ ), antiviral $\left({ }^{(L e e}\right.$ et al.. ${ }^{[8]}$ ), antianemic (Simsek, Karadeniz, Kalkan, Keles, \& Unal, ${ }^{[9]}$ ), and antioxidant activity (Karkos, Leong, Karkos, Sivaji, \& Assimakopoulos, $\left.{ }^{[10]}\right)$. All of these nutraceutical benefits are attributed to substances in Spirulina known as phycobiliproteins (C-phycocyanin, allophycocyanin, phycoerythrin and phycoerythrocyanin), which constitute their own protein complex in association with their linker polypeptides, called phycobilisome (Gantt, Lipschultz, Grabowski, \& Zimmerman, ${ }^{[11]}$; Hoseini, Khosravi-Darani, \& Mozafari, $\left.{ }^{[12]}\right)$. Isolation and testing of these phycobiliproteins has shown that they possess same beneficial effects as the whole microalgae. Hence, they are considered as the actual bioactive agents in this functional food (Eriksen, ${ }^{[13]}$; Hoseini et al., ${ }^{[12]}$; Khan et al., ${ }^{[14]}$ ).

\section{Obtention of C-phycocyanin: brief historical perspective}

Different methods of extraction, isolation and purification (summarized in Figure 1) have been assayed in order to obtain these phycobiliproteins (especially C-phycocyanin) from Spirulina spp (Glazer, Lundell, Yamanaka, \& Williams, ${ }^{[15]}$; Khan et al., ${ }^{[14]}$ ). The first attempts involved simple chromatography by using precipitations previously obtained with ammonium sulphate. Subsequently, phycobiliproteins were isolated by crystallization (Carra, ${ }^{[16]}$ ). However, these methods lacked specificity as they extracted the whole phycobilisome without separating each pigment.

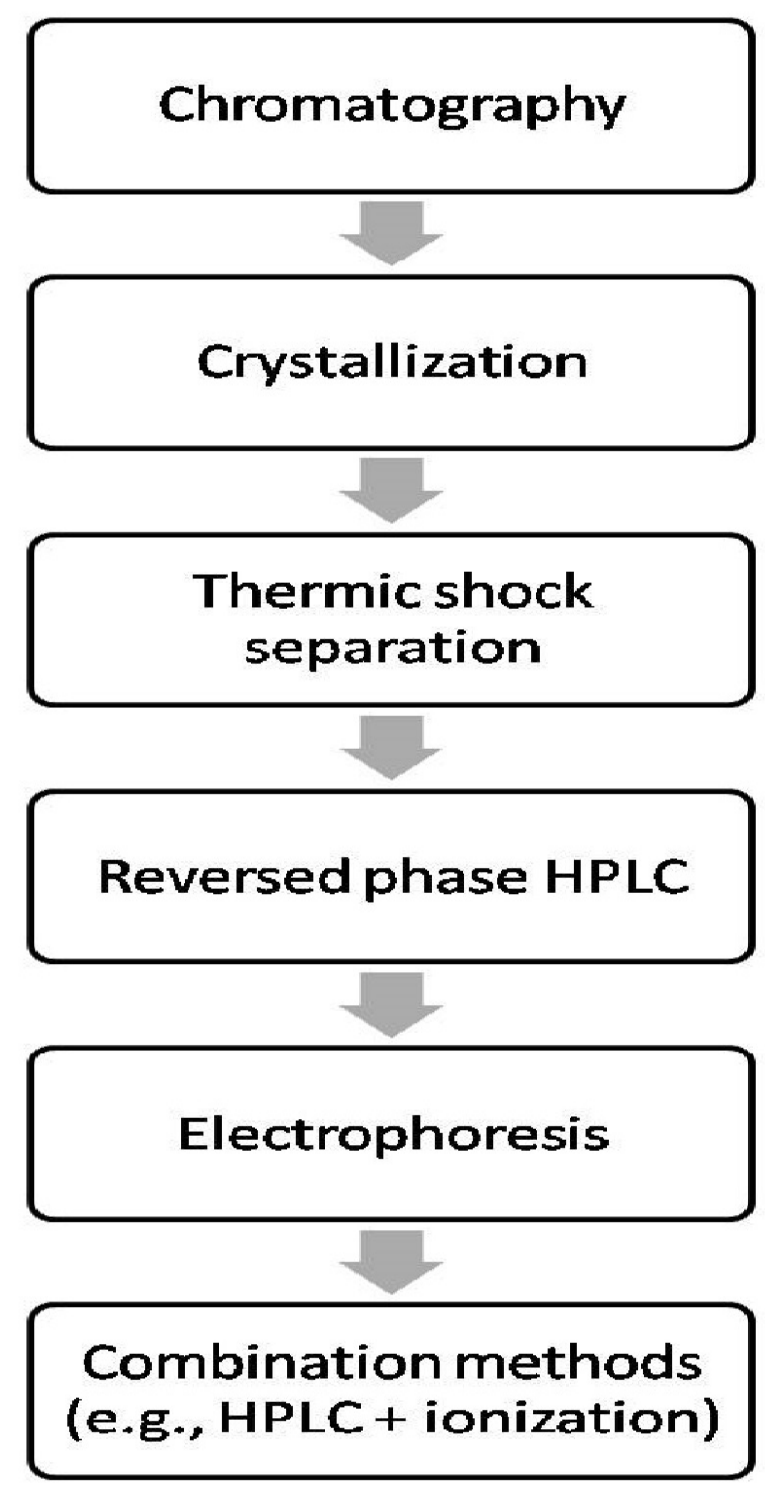


Years later, a thermal shock-based separation technique allowed pigment separation via density-gradient centrifugation with sucrose (Bekasova, Muslimov, \& Krasnovskiii, $\left.{ }^{[17]}\right)$. Although these processes represented an advance, the overall purity and yield of the process was still low.

With the use of reversed phase high-performance liquid chromatography (RP-HPLC), it was possible to achieve an isolation of up to $85 \%$ of C-phycocyanin and allophycocyanin. (Swanson \& Glazer, ${ }^{[18]}$ ) Nevertheless, other compounds apart from the phycobilisome were present in the final products due to the sample pre-treatments needed to perform HPLC. In order to optimize phycobiliprotein isolation, the chromatographic method was modified by adapting resins used in the solid phase, varying the polarity and $\mathrm{pH}$ of the eluent solution (Moreno et al., ${ }^{[19]}$ ), and using magnesium chloride precipitation with further diffusion in polyethylene glycol gel. By enhancing overall specificity, the isolation of pure C-phycocyanin and allophycocyanin was obtained, but the yielding mass was significantly decreased.

Towards the end of the $20^{\text {th }}$ century, electrophoresis-based techniques were tested with the addition of laser-induced fluorescence (LIF) detectors. This novel achieved separation with a fairly good yield $(\approx 90-100 \%)$. Nevertheless, such extractions corresponded to a mixture of both C-phycocyanin and allophycocyanin (Viskari \& Colyer, ${ }^{[20]}$ ). Yet another type of electrophoresis was used with polyacrylamide/dodecyl sulphate gel, pre-treating the samples by precipitation with ammonium sulphate followed by further separation in chromatographic columns by Sephadex (Minkova et al., 2003). This technique achieved an isolation of pure C-phycocyanin with a yield of $\approx 45 \%$.

Afterwards, different combinations of methods were tested in order to increase the ease of separation and isolation as well as the grade of purity and final yield. One such method, HPLC coupled with a flame ionization detector (Zolla \& Bianchetti, ${ }^{[2]}$ ), was able to separate $\mathrm{C}$-phycocyanin from allophycocyanin, yet it destroyed the original sample. In order to resolve this problem, an integral procedure was devised that extracted the phycobiliproteins with sodium phosphate neutral buffer, and then further purified them via dialysis and gel filtration chromatography (Bhaskar, Gopalaswamy, \& Raghu ${ }^{[22]}$ ). This procedure yielded C-phycocyanin with a purity of 4.98. Another method, pre-treated the sample in the same manner, but the purification process consisted of ion-exchange chromatography (Patel, Mishra, Pawar, \& Ghosh, ${ }^{[23]}$ ), yielding C-phycocyanin with a purity of 4.42.

Another widely studied method for isolating C-phycocyanin from Spirulina platensis combined chromatography with expanded bed adsorption, anion interchange, and hydroxyapatite columns (Niu, Wang, Lin, \& Zhou, $\left.{ }^{[24}\right)$. These techniques yielded $4.45 \mathrm{mg}$ of C-phycocyanin per gram of dried S. platensis with a purity of 3.2. This method offers several advantages, such as the possibility of using different resins with special charge characteristics (i.e., anionic or cationic). For instance, by using Q-Sepharose (Silveira, de Menezes Quines, Burkert, \& Kalil, 2008) it was possible to isolate C-phycocyanin from Spirulina platensis with $75 \%$ yield and a purity of 3.4. The problem with these kinds of techniques is that they are often strongly dependent on the $\mathrm{pH}$ and temperature of the eluent solutions.

On the other hand, hydrophobic interaction chromatography with ammonium sulphate and liquid nitrogen precipitation pretreatments (Soni, Trivedi, \& Madamwar, ${ }^{[25]}$ ) are capable of isolating C-phycocyanin with a purity of 4.5 but with poor yields. This may be due to the original cyanobacterium (Phormidium fragile) from which the phycobilin was isolated. To improve the yield, C-phycocyanin was extracted from Spirulina platensis with high-speed counter-current chromatography (HSCCC) (Yin et al., ${ }^{[26]}$ ), obtaining $78.7 \mathrm{mg}$ per $200 \mathrm{mg}$ of crude extract with a purity of 4.25 . Nowadays, one of the most widely used methods is ionic exchange chromatography, which involves pre treating vegetable samples of Spirulina platensis with two aqueous phases(Patil, Chethana, Sridevi, \& Raghavarao, $\left.{ }^{[27]}\right)$, leading to a purity of 6.69 .

The aim of the present review was to describe different methods for C-phycocyanin extraction and purification and compare the results in order to determine the method with the best cost-benefit ratio.

\section{Methods}

We performed an exhaustive search (using Scopus and PubMed databases) to find methods for the separation, isolation and purification of C-phycocyanin from different microorganisms. Search words were: extraction, separation, isolation and purification of C-phycocyanin.

\section{Results}

Summaries and general characteristics are herein presented (see Table) for the 86 reports found.

\begin{tabular}{|l|l|l|l|}
\hline $\begin{array}{l}\text { Extraction by } \\
\text { dell } \\
\text { disruption }\end{array}$ & Purification method & Observations & Reference \\
\hline $\begin{array}{l}\text { Thermal treat- } \\
\text { ment }\end{array}$ & Density gradient by centrifugation & $\begin{array}{l}\text { The phycobilisomes of } N \text {. muscorum were sepa- } \\
\text { rated into two subunits containing C-phycocyanin } \\
\text { and allophycocyanin. However, they had traces of } \\
\text { phycoerythrocyanin, thus presenting low purity } \\
\text { and low yields (data not shown). }\end{array}$ & Bekasova et al. $^{[17]}$ \\
\cline { 2 - 5 } & $\begin{array}{l}\text { Reverse phase chromatography using dicarbox- } \\
\text { ylic acids and methanol-butanol washes }\end{array}$ & $\begin{array}{l}\text { Traces of C-phycocyanin and phycoerythrocyanin } \\
\text { were identified by mass spectrometry chromatog- } \\
\text { raphy. }\end{array}$ & $\begin{array}{l}\text { Fu, Friedman, and } \\
\text { Siegelman }\end{array}$ \\
\hline
\end{tabular}


Extraction, Isolation and Purification of C-phycocyanin

\begin{tabular}{|c|c|c|c|c|c|}
\hline \multirow[t]{6}{*}{$\begin{array}{l}\text { Pressure ho- } \\
\text { mogenization }\end{array}$} & \multirow[t]{3}{*}{$\begin{array}{l}\text { Aqueous two- } \\
\text { phase system } \\
\text { (ATPS) }\end{array}$} & \multicolumn{2}{|c|}{$\begin{array}{l}\text { Ionic exchange chromatog- } \\
\text { raphy }\end{array}$} & $\begin{array}{l}\text { C-phycocyanin was obtained at a } 6.69 \text { grade of } \\
\text { purity from the aqueous extract of Spirulina } \\
\text { platensis. }\end{array}$ & Patil et al. ${ }^{[29]}$ \\
\hline & & \multicolumn{2}{|c|}{$\begin{array}{l}\text { Polyethylene glycol } 4000 \text { and } \\
\text { potassium phosphate satura- } \\
\text { tion }\end{array}$} & $\begin{array}{l}\text { C-phycocyaninwas obtained from Spirulina } \\
\text { platensis in a single extraction step. With multiple } \\
\text { extractions, the purity of the isolates increases } \\
\text { from } 3.23 \text { to } 4.02 \text {. }\end{array}$ & $\begin{array}{l}\text { Patil, Chethana, } \\
\text { Madhusudhan, and } \\
\text { Raghavarao }^{[29]}\end{array}$ \\
\hline & & $\begin{array}{l}\text { Ultra } \\
\text { filtration }\end{array}$ & $\begin{array}{l}\text { Salting out } \\
\text { (precipitation } \\
\text { crystallization) }\end{array}$ & $\begin{array}{l}\text { C-phycocyaninwas obtained from Spirulina maxi- } \\
m a \text { with a purity of } 3.8 \% .\end{array}$ & $\begin{array}{l}\text { Rito-Palomares, } \\
\text { Nuñez, and Ama- } \\
\text { dor }^{[30]}\end{array}$ \\
\hline & $\begin{array}{l}\text { Hexane ex- } \\
\text { traction }\end{array}$ & \multicolumn{2}{|c|}{ SDS-PAGE electrophoresis } & $\begin{array}{l}\text { C-phycocyanin was obtained from Spirulina spp } \\
\text { at a yield of } 10.2 \% \text { and a purity of } 1 .\end{array}$ & Seo et al. ${ }^{[31]}$ \\
\hline & $\begin{array}{l}\text { Stirring-centrif- } \\
\text { ugation }\end{array}$ & \multicolumn{2}{|c|}{$\begin{array}{l}\text { Expanded bed anion exchange } \\
\text { with } 80 \% \text { ammonium sulfate }\end{array}$} & $\begin{array}{l}25.7 \mathrm{mg} \mathrm{g}^{-1} \mathrm{dm} \text { of C-phycocyanin was obtained } \\
\text { from fresh Spirulina platensis at a purity of } 4.8 \text {. }\end{array}$ & $\begin{array}{l}\text { Moraes, Mazutti, } \\
\text { Maugeri, and Kalil } \\
{[32]}\end{array}$ \\
\hline & & \multicolumn{2}{|c|}{$\begin{array}{l}\text { Precipitation with ammonium } \\
\text { sulfate Fast flow chromatog- } \\
\text { raphy DEAE-Sepharose and } \\
\text { hydroxyapatite columns }\end{array}$} & $\begin{array}{l}\text { C-phycocyanin was isolated from Spirulina } \\
\text { platensis with a yield of } 30 \mathrm{mg} \mathrm{g}^{-1} \mathrm{dm} \text { and a purity } \\
\text { of } 3.94 \text {. }\end{array}$ & $\begin{array}{l}\text { Ou, Lin, Yang, Pan, } \\
\text { and Cheng }{ }^{[33]}\end{array}$ \\
\hline \multirow{6}{*}{$\begin{array}{l}\text { Freeze-un- } \\
\text { freeze } \\
\text { agitation }\end{array}$} & \multirow{4}{*}{$\begin{array}{l}\text { Aqueous two- } \\
\text { phase system } \\
\text { (ATPS) }\end{array}$} & \multicolumn{2}{|c|}{ Gel filtration chromatography } & $\begin{array}{l}\text { C-phycocyaninwas obtained from Spirulina maxi- } \\
m a \text { with a yield of } 46.5 \% \text { and a purity of } 3.4 .\end{array}$ & \multirow[t]{3}{*}{ Cruz de Jesús ${ }^{[34]}$} \\
\hline & & \multicolumn{2}{|c|}{ Ion exchange chromatography } & $\begin{array}{l}\text { C-phycocyanin was obtained from Spirulina } \\
\text { maxima with a yield of } 37.5 \% \text { and a purity of } 3.5 \\
\text { (determined by DEAE-Cellulose). }\end{array}$ & \\
\hline & & \multicolumn{2}{|c|}{ Ultra filtration } & $\begin{array}{l}\text { In this last stage, C-phycocyanin was isolated } \\
\text { from fresh Spirulina maxima with a yield of } 57 \% \\
\text { and a purity of } 3.9 \text {. }\end{array}$ & \\
\hline & & \multicolumn{2}{|c|}{ Ultracentrifugation } & $\begin{array}{l}\text { C-phycocyaninwas isolated from cyanobacteria } \\
\text { (Spirulina maxima and Porphyridium cruentum) } \\
\text { with a yield of } 98 \% \text {, but with low purity }(2.1) \text {. }\end{array}$ & $\begin{array}{l}\text { Benavides and Ri- } \\
\text { to-Palomares }{ }^{[35]}\end{array}$ \\
\hline & $\begin{array}{l}\text { Organic sol- } \\
\text { vents and buf- } \\
\text { fer extraction }\end{array}$ & \multicolumn{2}{|c|}{ Centrifugation and filtration } & $\begin{array}{l}\text { C-phycocyanin obtained from Spirulina platensis } \\
\text { had low yield and low purity }(0.46) .\end{array}$ & $\begin{array}{l}\text { Silveira, Burkert, } \\
\text { Costa, Burkert, and } \\
\text { Kalil }^{[36]}\end{array}$ \\
\hline & & \multicolumn{2}{|c|}{$\begin{array}{l}\text { Polyethylene glycol systems } \\
1500,4000 \text { and } 6000 / \text { aqueous } \\
\text { two-phase system (ATPS) }\end{array}$} & $\begin{array}{l}2.67 \mathrm{mg} / \mathrm{g} \text { of C-phycocyanin was isolated from } \\
\text { Spirulina platensis with a purity of } 0.79 .\end{array}$ & $\begin{array}{l}\text { Antelo, Anschau, } \\
\text { Costa, and Kalil }{ }^{[37]}\end{array}$ \\
\hline \multirow[t]{6}{*}{$\begin{array}{l}\text { Freeze-un- } \\
\text { freeze cycles }\end{array}$} & \multicolumn{3}{|c|}{ Agitation-centrifugation } & $\begin{array}{l}\text { C-phycocyanin was extracted and isolated from } \\
\text { some cyanobacteria (Synechocystisspp, Glueuco- } \\
\text { psaspp, Anabaena spp and Lyngbyaspp) with a } \\
\text { yield of } 100 \mu \mathrm{g} / \mathrm{g} \mathrm{dm} \text { and a purity of } 3.1 \text {. }\end{array}$ & $\begin{array}{l}\text { Maurya, Maurya, } \\
\text { and Pandey }{ }^{[38]}\end{array}$ \\
\hline & \multirow[t]{3}{*}{ Ultra filtration } & \multirow{2}{*}{\multicolumn{2}{|c|}{ Ion exchange chromatography }} & $\begin{array}{l}\text { C-phycocyanin was isolated from P. ceylanicum } \\
\text { with a yield of } 63.50 \% \text { anda purity of } 4.15 \text {. }\end{array}$ & $\begin{array}{l}\text { Singh, Parmar, and } \\
\text { Madamwar (2009) }\end{array}$ \\
\hline & & & & $\begin{array}{l}\text { A phycobiliprotein was obtained fromthe fresh } \\
\text { biomass of Spirulina spp witha yield of } 82.9 \text { to } \\
88.6 \% \text { and a purity of } 1.0 \text {. }\end{array}$ & $\begin{array}{l}\text { Chaiklahan, Chirasu- } \\
\text { wan, Loha, Tia, and } \\
\text { Bunnag }{ }^{[39]}\end{array}$ \\
\hline & & \multicolumn{2}{|c|}{$\begin{array}{l}\text { Tri chloro acetic precipitation } \\
\text { (TCA)/centrifugation } \\
\text { Electrophoresis } \\
\text { SDS-PAGE }\end{array}$} & $\begin{array}{l}\text { C-phycocyanin was isolated from cyano bacteria } \\
\text { Phorphyra columbina with a yield of } 19.9 \mathrm{mg} / \mathrm{g} \\
\mathrm{dm} \text { and a purity of } 0.08 .\end{array}$ & $\begin{array}{l}\text { Cian, López-Posa- } \\
\text { das, Drago, Medina, } \\
\text { and Martínez-Augus- } \\
\operatorname{tin}^{[40]}\end{array}$ \\
\hline & \multirow[t]{2}{*}{$\begin{array}{l}\text { Cell disruption } \\
\text { by pressure / } \\
\text { agitation and } \\
\text { centrifugation }\end{array}$} & \multirow{2}{*}{\multicolumn{2}{|c|}{$\begin{array}{l}\text { Purification by hydroxyapatite } \\
\text { column chromatography and } \\
\text { anion exchange / ultra filtra- } \\
\text { tion / electrophoresis } \\
\text { SDS-PAGE }\end{array}$}} & $\begin{array}{l}\text { C-phycocyanin was extracted from the cyano } \\
\text { bacteria Anabaena spp with a yield of } 10 \% \text { and a } \\
\text { purity of } 2.7 \text {. }\end{array}$ & $\begin{array}{l}\text { Ducret, Sidler, } \\
\text { Wehrli, Frank, and } \\
\text { Zuber }^{[41]}\end{array}$ \\
\hline & & & & $\begin{array}{l}\text { C-phycocyaninwas isolated from Spirulina spp } \\
\text { and purified, with a yield of } 85 \% \text { and a purity of } \\
3.66 \text {. }\end{array}$ & $\begin{array}{l}\text { Yoshida, Takagaki, } \\
\text { and Nishimune }{ }^{[42]}\end{array}$ \\
\hline
\end{tabular}




\begin{tabular}{|c|c|c|c|}
\hline \multirow{14}{*}{$\begin{array}{l}\text { Precipitation } \\
\text { with ammo- } \\
\text { nium sulfate } \\
(25 \%)\end{array}$} & \multirow{6}{*}{$\begin{array}{l}\text { Column elution hydroxyap- } \\
\text { atite / Sephadex -DEAE ion } \\
\text { exchange / Bio-Gel electro- } \\
\text { phoresis P }\end{array}$} & $\begin{array}{l}\text { C-phycocyanin was extracted from lyophilized } \\
\text { Spirulina platensis with a purity of } 4.0 .\end{array}$ & $\begin{array}{l}\text { Bermejo-Bescós, } \\
\text { Piñero-Estrada, and } \\
\text { Villar del Fresno }^{[43]}\end{array}$ \\
\hline & & $\begin{array}{l}\text { C-phycocyanin was isolated from Synechococcus } \\
\text { spp and Aphanocapsa cyano bacteria with a yield } \\
\text { of } 50 \% \text { and a purity of } 6.1 .\end{array}$ & $\begin{array}{l}\text { Glazer and Co- } \\
\text { hen-Bazire }^{[44]}\end{array}$ \\
\hline & & $\begin{array}{l}\text { C-phycocyaninwas extracted and purified from } \\
\text { Porphyrayezoensis cyano bacterium with a yield } \\
\text { of } 20 \% \text { and a purity of } 0.9\end{array}$ & $\mathrm{He}, \mathrm{Hu}$, and Jiang ${ }^{[45]}$ \\
\hline & & $\begin{array}{l}\text { C-phycocyanin was isolated and purified from the } \\
\text { fresh biomass of Spirulina platensis with a yield } \\
\text { of } 13.1 \% \text { and a purity of } 4.71 \text {. }\end{array}$ & $\begin{array}{l}\mathrm{Li} \text {, Zhang, Gao, and } \\
\mathrm{Chu}^{[46]}\end{array}$ \\
\hline & & $\begin{array}{l}\text { C-phycocyanin was extracted and isolated from } \\
\text { Spp Chroomonas cyano bacterium with a yield of } \\
59 \% \text { and a purity of } 0.92 \text {. }\end{array}$ & $\begin{array}{l}\text { MacColl, Habig, and } \\
\text { Berns }^{[47]}\end{array}$ \\
\hline & & $\begin{array}{l}\text { C -phycocyanin was isolated from fresh Spirulina } \\
\text { platensis with a yield of } 95 \mu \mathrm{g} / \mathrm{g} \text { dmand a purity } \\
\text { of } 3.9 .\end{array}$ & $\begin{array}{l}\text { Piñero Estrada, } \\
\text { Bermejo Bescós, and } \\
\text { Villar del Fresno }^{[48]}\end{array}$ \\
\hline & \multirow[t]{2}{*}{$\begin{array}{l}\text { Step chromatography with } \\
\text { DEAE cellulose- } 11\end{array}$} & $\begin{array}{l}\text { The extract isolated from Spirulina platensis was } \\
\text { identified as C-phycocyaninby SDS-PAGE, with } \\
\text { a yield of } 80 \% \text { and a purity of } 4.5 \text {. }\end{array}$ & $\begin{array}{l}\text { Kumar, Dhar, Pabbi, } \\
\text { Kumar, and Walia }^{[49]}\end{array}$ \\
\hline & & $\begin{array}{l}\text { C-phycocyanin was extracted and isolated from } \\
\text { Anabaena variabilis cyano bacterium with a yield } \\
\text { of } 36 \% \text { and a purity of } 2.75 \text {. }\end{array}$ & $\begin{array}{l}\text { Chakdar, Saha, and } \\
\text { Pabbi }^{[50]}\end{array}$ \\
\hline & \multirow[t]{4}{*}{$\begin{array}{l}\text { Precipitation with ammonium } \\
\text { sulfate }(50 \%)\end{array}$} & $\begin{array}{l}\text { C-phycocyanin was obtained from Galdieria } \\
\text { sulphuraria cyano bacteria with a yield of } 80 \% \\
\text { and a purity of } 4 \text {. }\end{array}$ & Moon et al. ${ }^{[51]}$ \\
\hline & & $\begin{array}{l}\text { C-phycocyanin was isolated and purified from } \\
\text { Spirulina maxima with yield of } 24 \% \text { and a purity } \\
\text { of } 2.25 \text {. }\end{array}$ & $\begin{array}{l}\text { Abd El-Baky and } \\
\text { El-Baroty }{ }^{[52]}\end{array}$ \\
\hline & & $\begin{array}{l}\text { C-phycocyaninwas extracted and isolated from } \\
\text { cyanobacterium of the Nostoc spp genus, with a } \\
\text { yield of } 59 \% \text { and a purity of } 2.8 \text {. }\end{array}$ & $\begin{array}{l}\text { Gray, Lipschultz, and } \\
\text { Gantt }^{[53]}\end{array}$ \\
\hline & & $\begin{array}{l}\text { C-phycocyaninwas isolated and purified from } \\
\text { Spirulina fusiformis with a yield of } 60 \% \text { and } \\
\text { purity of } 3.8 \text {. }\end{array}$ & $\begin{array}{l}\text { Madhyastha, Radha, } \\
\text { Sugiki, Omura, and } \\
\text { Maruyama }^{[54]}\end{array}$ \\
\hline & Ultracentrifugation & $\begin{array}{l}\text { C-phycocyanin was isolated from Cyanidium } \\
\text { caldarium cyanobacterium with a yield of } 15 \mathrm{mg} \\
\text { g-1dmand a purity of } 7 .\end{array}$ & $\begin{array}{l}\text { Stec, Troxler, and } \\
\text { Teeter }^{[55]}\end{array}$ \\
\hline & $\begin{array}{l}\text { Activated carbon and chitosan } \\
\text {, flow filtration }\end{array}$ & $\begin{array}{l}\text { High purity C- phycocyanin was obtained from } \\
\text { Limnotrix spp with low ammonium sulfate con- } \\
\text { centrations. }\end{array}$ & $\begin{array}{l}\text { Gantar, Simović, } \\
\text { Djilas, Gonzalez, and } \\
\text { Miksovska }^{[56]}\end{array}$ \\
\hline $\begin{array}{l}\text { Ionic Exchange } \\
\text { chromatog- } \\
\text { raphy (DE- } \\
\text { AE-Sephadex) }\end{array}$ & & $\begin{array}{l}\text { A phycobiliproteinwas obtained from Spirulina } \\
\text { platensis under a three-step procedure, increasing } \\
\text { its purity to } 4.3 \text { (identified by SDS-PAGE). }\end{array}$ & $\begin{array}{l}\text { Liao, Zhang, Wang, } \\
\text { Yan, and Zhang }{ }^{[57]}\end{array}$ \\
\hline $\begin{array}{l}\text { Anion } \\
\text { exchange chro- } \\
\text { matography by } \\
\text { hydrophobic } \\
\text { interactions ( } \\
\text { butyl- Sephar- } \\
\text { ose column ) }\end{array}$ & $\begin{array}{l}\text { Ion exchange chromatography } \\
\text { (Q-Sepharose column) / filtra- } \\
\text { tion SDS-PAGE gel }\end{array}$ & $\begin{array}{l}\text { C-phycocyaninwas obtained from Synechococcus } \\
\text { spp with high purity and good yield. }\end{array}$ & $\begin{array}{l}\text { Abalde, Berancour, } \\
\text { Torres, Cid, and } \\
\text { Barwell }{ }^{[58]}\end{array}$ \\
\hline \multicolumn{2}{|c|}{ Tricalcium phosphate gel chromatography } & $\begin{array}{l}\text { A set of phycobiliproteins was extracted from } \\
\text { Smithoranaiadum microalga. Allophycocyanin, } \\
\text { phycoerythrocyanin and C- phycocyanin were } \\
\text { obtained after centrifugation, the latter at a low } \\
\text { yield. }\end{array}$ & $\begin{array}{l}\text { ÓhEocha and } \\
\operatorname{Haxo}^{[59]}\end{array}$ \\
\hline
\end{tabular}


Extraction, Isolation and Purification of C-phycocyanin

\begin{tabular}{|c|c|c|c|c|}
\hline Cellular & \multicolumn{2}{|c|}{ Purification method } & \multirow{2}{*}{$\begin{array}{l}\text { Observations } \\
\begin{array}{l}\text { A mixture of C-phycocyanin and R- phycocyanin- } \\
\text { phycoblasts were obtained from N. muscorum. }\end{array}\end{array}$} & \multirow{2}{*}{$\begin{array}{l}\text { References } \\
\text { Carra }^{[16]}\end{array}$} \\
\hline \multirow[t]{20}{*}{$\begin{array}{l}\text { Liquid } \\
\text { nitrogen }\end{array}$} & \multirow[t]{4}{*}{$\begin{array}{l}\text { Precipitation/ } \\
\text { crystallization }\end{array}$} & Ammonium sulfate & & \\
\hline & & $\begin{array}{l}\text { Ammonium-sulfate / hydro- } \\
\text { phobic interaction chromatog- } \\
\text { raphy }\end{array}$ & $\begin{array}{l}\text { C-phycocyanin was isolated from Phormidium } \\
\text { fragile, with low yield and a purity of } 4.52 \text {. }\end{array}$ & Soni et al. ${ }^{[25]}$ \\
\hline & & $\begin{array}{l}\text { SDS-PAGE and mercaptoetha- } \\
\text { nol / chromatography (column } \\
\text { sulfonated polystyrene) }\end{array}$ & $\begin{array}{l}\text { Allophycocyanin and C-phycocyanin were isolat- } \\
\text { ed among Phormidiumluridum phycobili proteins, } \\
\text { with low yield and low purity. }\end{array}$ & $\begin{array}{l}\text { Kobayashi, Siegel- } \\
\text { man, and Hirs }{ }^{[60]}\end{array}$ \\
\hline & & $\begin{array}{l}\text { Stirring and precipitation with } \\
\text { ammonium sulfate / SDS- } \\
\text { PAGE electrophoresis }\end{array}$ & $\begin{array}{l}\text { C-phycocyanin was isolated from Oscillatona } \\
\text { cyanobacterial agardhii with } 70 \% \text { yield and a } \\
\text { purity of } 4.35 \text {. }\end{array}$ & $\begin{array}{l}\text { Torjesen and Slet- } \\
\text { ten }^{[61]}\end{array}$ \\
\hline & \multirow{2}{*}{$\begin{array}{l}\text { High perfor- } \\
\text { mance liquid } \\
\text { chromatogra- } \\
\text { phy (HPLC ) }\end{array}$} & Reversed phase & $\begin{array}{l}\text { C-phycocyanin and allophycocyan in were isolat- } \\
\text { ed with a yield of } 85 \% \text {. The impurities were traces } \\
\text { of compounds outside the phycobilisome. }\end{array}$ & $\begin{array}{l}\text { Swanson and Glaz- } \\
\mathrm{er}^{[18]}\end{array}$ \\
\hline & & Flame ionization & $\begin{array}{l}\text { C-phycocyanin was separated from allophycocy- } \\
\text { anin(derived from Synechocystis). However, in the } \\
\text { identification process the sample was lost. }\end{array}$ & $\begin{array}{l}\text { Zolla and Bianchetti } \\
2001\end{array}$ \\
\hline & \multirow[t]{3}{*}{$\begin{array}{l}\text { SDS-PAGE- } \\
\text { electrophoresis }\end{array}$} & $\begin{array}{l}\text { Laser induced fluorescence } \\
\text { (LIF ) }\end{array}$ & $\begin{array}{l}\text { A mixture of C-phycocyanin and allophycocyanin } \\
\text { was extracted, with yields of about 93-105\%. }\end{array}$ & Viskari and Colyer ${ }^{[20]}$ \\
\hline & & $\begin{array}{l}\text { Polyacrylamide gel dodecyl } \\
\text { sulfate / Sephadex column }\end{array}$ & $\begin{array}{l}\text { C-phycocyanin was extracted from Spirulina } \\
\text { fusiformis with a } 46 \% \text { yield. }\end{array}$ & $\begin{array}{l}\text { Minkova et al. } \\
(2003)\end{array}$ \\
\hline & & $\begin{array}{l}\text { Freezing and thawing cycles } \\
\text { / dialysis / centrifugation / } \\
\text { ammonium sulfate }(20 \%) \\
\text { precipitation, and chromatog- } \\
\text { raphy by filtration on DEAE } \\
\text { - Sepharose gel }\end{array}$ & $\begin{array}{l}\text { C-phycocyanin was obtained from cyano bacteri- } \\
\text { um Oscillatoria tenuis with a yield of } 61.8 \% \text { and } \\
\text { a purity of } 4.88 \text {. }\end{array}$ & Thangam et al. ${ }^{[62]}$ \\
\hline & $\begin{array}{l}\text { Chromatogra- } \\
\text { phy }\end{array}$ & $\begin{array}{l}\text { Magnesium chloride / polyeth- } \\
\text { ylene glycol } 6000\end{array}$ & $\begin{array}{l}\text { A mixture of C-phycocyanin and allophycocyanin } \\
\text { was obtained from Spirulina platensis with low } \\
\text { yield and high purity, analyzed by X-ray diffrac- } \\
\text { tion. }\end{array}$ & Moreno et al. ${ }^{[19]}$ \\
\hline & & $\begin{array}{l}\text { Gel filtration / ammonium } \\
\text { sulfate / dialysis }\end{array}$ & $\begin{array}{l}\text { C-phycocyanin was obtained from Spirulina plat- } \\
\text { ensis at a purity of } 4.98 \text {. }\end{array}$ & Bhaskar et al. ${ }^{[22]}$ \\
\hline & & \multirow[t]{2}{*}{ Ionic exchange } & $\begin{array}{l}\text { C-phycocyanin was extracted from Spirulina } \\
\text { with a purity of } 4.42 .\end{array}$ & Patel et al. ${ }^{[23]}$ \\
\hline & & & $\begin{array}{l}\text { C-phycocyanin was isolated from } S . \text { platensis- } \\
\text { using Q-Sepharose, with a yield of } 77.3 \% \text { and a } \\
\text { purity of } 3.4 \text {. }\end{array}$ & Silveira et al. (2008) \\
\hline & & $\begin{array}{l}\text { Hydrophobic interaction chro- } \\
\text { matography / ion exchange } \\
\text { chromatography }\end{array}$ & $\begin{array}{l}\text { C-phycocyanin was extracted from Calothrixsp } \\
\text { with apurity of } 3.3 \text {. }\end{array}$ & $\begin{array}{l}\text { Santiago-Santos, } \\
\text { Ponce-Noyola, } \\
\text { Olvera-Ramírez, } \\
\text { Ortega-López, and } \\
\text { Cañizares-Villanue- } \\
\text { va }^{[63]}\end{array}$ \\
\hline & & \multirow{4}{*}{$\begin{array}{l}\text { Bed adsorption / anion } \\
\text { exchange / hydroxyapatite } \\
\text { column }\end{array}$} & $\begin{array}{l}4.45 \mathrm{mg} \text { g- } 1 \text { of C-phycocyanin was isolated from } \\
\text { dry } S \text {. platensis with a purity of } 3.2 \text {. }\end{array}$ & Niu et al. ${ }^{[24]}$ \\
\hline & & & $\begin{array}{l}\text { C-phycocyanin was isolated from cyanobacterium } \\
\text { Aphanizomenonflos-aquae with a purity of } 4.78 .\end{array}$ & Benedetti et al. ${ }^{[64]}$ \\
\hline & & & $\begin{array}{l}\text { C-phycocyanin was purified from Porphy- } \\
\text { rayezoensisby electrophoresis, with a good yield } \\
\text { and high grade of purity. }\end{array}$ & Cai et al. ${ }^{[65]}$ \\
\hline & & & $\begin{array}{l}\text { C-phycocyanin was isolated from Anabaena ma- } \\
\text { rine with a yield of } 62 \% \text { and a purity of } 4 \text {. }\end{array}$ & $\begin{array}{l}\text { Ramos, Acién, } \\
\text { Fernández-Sevilla, } \\
\text { González, and Ber- } \\
\text { mejo }^{[66]}\end{array}$ \\
\hline & & $\begin{array}{l}\text { Liquid phaseisoelectric } \\
\text { focusing }\end{array}$ & $\begin{array}{l}\text { C-phycocyanin was isolated from Spirulina plat- } \\
\text { ensis with a yield of } 39.2 \% \text { and a purity of } 4.0 \text {. }\end{array}$ & $\begin{array}{l}\text { Huang, Yang, Zheng, } \\
\text { and Guo }{ }^{[67]}\end{array}$ \\
\hline & & $\begin{array}{l}\text { High-speed counter current } \\
\text { chromatography (HSCCC) / } \\
\text { reversed phase }\end{array}$ & $\begin{array}{l}79 \text { mg of C-phycocyanin was extracted from } \\
\text { Spirulina platensis with a purity of } 4.25 \text {, and was } \\
\text { identified by SDS-PAGE. }\end{array}$ & Yin et $\mathrm{al}^{[26]}$ \\
\hline
\end{tabular}




\begin{tabular}{|c|c|c|c|c|}
\hline \multirow[t]{15}{*}{ Osmotic shock } & \multirow{4}{*}{$\begin{array}{l}\text { Bed adsorption } \\
\text { chromatogra- } \\
\text { phy ( stream- } \\
\text { line - DEAE } \\
\text { column ) }\end{array}$} & \multirow[t]{4}{*}{$\begin{array}{l}\text { Ion exchange chromatography } \\
\text { ( DEAE - cellulose ) }\end{array}$} & $\begin{array}{l}\text { C-phycocyaninwas isolated from Spirulina plat- } \\
\text { ensis with a purity of } 4.6 \text {. }\end{array}$ & $\begin{array}{l}\text { Moraes, da Costa } \\
\text { Ores, Costa, and } \\
\text { Kalil }^{[68]}\end{array}$ \\
\hline & & & $\begin{array}{l}\text { C-phycocyanin was isolated from cyanobacteri- } \\
\text { um Synechocystisaquatilis with a yield of } 74 \% \\
\text { and a purity of } 4.0 \text {. }\end{array}$ & $\begin{array}{l}\text { Ramos, Acién, } \\
\text { Fernández-Sevilla, } \\
\text { González, and Ber- } \\
\text { mejo }^{[69]}\end{array}$ \\
\hline & & & $\begin{array}{l}\text { C-phycocyanin was isolated from Spirulina } \\
\text { platensis with a yield of } 59 \% \text { and a high degree of } \\
\text { purity, andwas identified by SDS-PAGE. }\end{array}$ & $\begin{array}{l}\text { Ruperto Bermejo and } \\
\operatorname{Ramos}^{[70]}\end{array}$ \\
\hline & & & $\begin{array}{l}\text { C-phycocyanin was isolated from Spirulina } \\
\text { platensis with pharmaceutical purity (4), and was } \\
\text { identified by SDS-PAGE. }\end{array}$ & $\begin{array}{l}\text { R. Bermejo, Felipe, } \\
\text { Talavera, and Alva- } \\
\text { rez-Pez }{ }^{[71]}\end{array}$ \\
\hline & $\begin{array}{l}\text { Enzymatic } \\
\text { digestionwith } \\
\text { lysozyme }\end{array}$ & Activated carbon and chitosan & $\begin{array}{l}\text { C-phycocyanin was extracted from Synechococ- } \\
\text { cusspp with a yield of } 80 \% \text { and a purity of } 4.27 \text {. }\end{array}$ & Gupta and Sainis $^{[72]}$ \\
\hline & & $\begin{array}{l}\text { Dialysis / centrifugation/ } \\
\text { precipitation with ammonium } \\
\text { sulfate / ultrafiltration }\end{array}$ & $\begin{array}{l}\text { C-phycocyanin was extracted and isolated from } \\
\text { Coccochloris cyanobacterial elabens with a yield } \\
\text { of } 0.12 \mathrm{mg} \text { per gram of dry biomass and a purity } \\
\text { of } 2.5 \text {. }\end{array}$ & $\begin{array}{l}\text { Kao, Berns, and } \\
\text { Town }{ }^{[73]}\end{array}$ \\
\hline & & $\begin{array}{l}\text { Agitation / centrifugation / } \\
\text { precipitation with } 35 \% \text { am- } \\
\text { monium sulfate/ dialysis / fast } \\
\text { flow column chromatography } \\
\text { (DEAE-Sepharose ) }\end{array}$ & $\begin{array}{l}\text { C-phycocyanin was isolated from Spirulina } \\
\text { platensis with a yield of } 4.43 \mathrm{mg} \mathrm{g}^{-1} \text { of } \mathrm{dm} \text { and a } \\
\text { purity of } 3.9 .\end{array}$ & $\begin{array}{l}\text { Pleonsil, Soogarun, } \\
\text { and Suwanwong }\end{array}$ \\
\hline & & $\begin{array}{l}\text { Agitation / centrifugation / } \\
\text { precipitation with } 35 \% \text { and } \\
50 \% \text { of ammonium sulfate / } \\
\text { hydrophobic interaction Chro- } \\
\text { matography ( DEAE-Sephar- } \\
\text { ose column) / ion exchange } \\
\text { column/ chromatographic gel }\end{array}$ & $\begin{array}{l}\text { C-phycocyanin was isolated from fresh Spiruli- } \\
\text { na platensis with a yield of } 566.50 \mathrm{mg} / \mathrm{g}^{-1} \text { and a } \\
\text { purity of } 5.32 \text {. }\end{array}$ & $\begin{array}{l}\text { Song, Zhao, and } \\
\text { Wang }^{[75]}\end{array}$ \\
\hline & \multirow[t]{6}{*}{$\begin{array}{l}\text { Rivanol-am- } \\
\text { monium sulfate } \\
(50 \%) \text { precipi- } \\
\text { tation }\end{array}$} & $\begin{array}{l}\text { Activated carbon and chitosan } \\
\text { / purification by Sephadex } \\
\text { column }\end{array}$ & $\begin{array}{l}\text { C-phycocyanin was isolated from two species of } \\
\text { cyanobacteria (Spirulina maximum and Spirulina } \\
\text { fusiformis) with a yield of } 55 \% \text { and a purity of } \\
4.50 \text { in both species, and was identified by SDS- } \\
\text { PAGE. }\end{array}$ & $\begin{array}{l}\text { Kaledona Minkova } \\
\text { et al. }{ }^{[76]}\end{array}$ \\
\hline & & $\begin{array}{l}\text { Sodium chloride precipitation/ } \\
\text { Sephadex column purification }\end{array}$ & $\begin{array}{l}\text { C-phycocyanin was obtained from cyanobacte- } \\
\text { rium Africanum Arthronema with a yield of } 55 \\
\% \text { and a purity of } 4.52 \text {, and was identified by } \\
\text { SDS-PAGE. }\end{array}$ & K. Minkova et al. ${ }^{[77]}$ \\
\hline & & \multirow[t]{4}{*}{$\begin{array}{l}\text { Ion exchange chromatography } \\
\text { ( DEAE -Sepharose column ) } \\
\text { / filtration }\end{array}$} & $\begin{array}{l}\text { C-phycocyanin was isolated from cyanobacterium } \\
\text { Spirulina platensis, which was grown in medium } \\
\text { enriched with selenium, achieving a purity of } \\
5.12 \text {. }\end{array}$ & $\begin{array}{l}\text { Chen, Wong, and } \\
\text { Zheng }^{[78]}\end{array}$ \\
\hline & & & $\begin{array}{l}\text { C-phycocyanin was isolated from Spirulina plat- } \\
\text { ensis after two purification processes with a yield } \\
\text { of } 67.04 \% \text { and a purity of } 5.59 \text {. }\end{array}$ & Yan et al. ${ }^{[79]}$ \\
\hline & & & $\begin{array}{l}\text { C-phycocyanin was isolated from Spirulina plat- } \\
\text { ensis cyano bacterium with a purity of } 4.0 \text {. }\end{array}$ & Moraes and Kalil ${ }^{[80]}$ \\
\hline & & & $\begin{array}{l}\text { C-phycocyanin was isolated from Spirulina plat- } \\
\text { ensis with pharmaceutical grade purity }(5.06) \text {. }\end{array}$ & Zhang and Chen ${ }^{[81]}$ \\
\hline & $\begin{array}{l}\text { Aqueous two- } \\
\text { phase system } \\
\text { (ATPS ) }\end{array}$ & $\begin{array}{l}\text { Ultrafiltration dialysis mem- } \\
\text { brane / ion exchange chroma- } \\
\text { tography (DEAE -Sepharose } \\
\text { column) }\end{array}$ & $\begin{array}{l}\text { C-phycocyanin was isolated from Galdieria sul- } \\
\text { phuraria with a yield of } 42 \% \text { and a purity of } 4.5 \text {. }\end{array}$ & $\begin{array}{l}\text { Sørensen, Hantke, } \\
\text { and Eriksen }{ }^{[82]}\end{array}$ \\
\hline & $\begin{array}{l}\text { EDTA precip- } \\
\text { itation / filtra- } \\
\text { tion / agitation / } \\
\text { centrifugation }\end{array}$ & SDS-PAGE electrophoresis & $\begin{array}{l}\text { C-phycocyanin was extracted and isolated from } \\
\text { Acaryochloris marine cyanobacterium with a } \\
\text { yield of } 15 \% \text { and a purity of } 2.0 \text {. }\end{array}$ & $\begin{array}{l}\text { Marquardt, Senger, } \\
\text { Miyashita, Miyachi, } \\
\text { and Mörschel }{ }^{[83]}\end{array}$ \\
\hline & $\begin{array}{l}\text { Ultrasonication } \\
\text { with buffer }\end{array}$ & & $\begin{array}{l}\text { C-phycocyanin was isolated from Spirulina plat- } \\
\text { ensis with pharmaceutical grade purity. }\end{array}$ & $\begin{array}{l}\text { Sun, Wang, and } \\
\text { Qiao }^{[84]}\end{array}$ \\
\hline
\end{tabular}




\section{Discussion}

Novel extraction, isolation, and purification processes for C-phycocyanin have been sought and developed since the 1980's. (Khan et al., ${ }^{[73]}$ ) Throughout this process, it has been demonstrated that the original biomass is of critical importance in order to reach the best cost-benefit ratio when isolating phycobiliproteins. Another feature that must be considered is the freshness of the biomass and the subsequent pretreatment processes. In this series, C-phycocyanin was obtained from fresh biomass and dried at room temperature (as opposed to using lyophilized powders) (Chaiklahan et al., ${ }^{[15]} 2011$; Niu et al., ${ }^{[50]}$ ).

Regarding extraction and purification methods, previous studies have shown that multiple cycles improved the purity grade of the C-phycocyanin extract, although yields significantly decreased. For instance, an aqueous two-phase system with polyethylene glycol 4000 (Patil et al., ${ }^{[4]}$ ) managed to increase purity, but yields were importantly reduced. Similarly, a multiple extraction process for obtaining C-phycocyanin by using a Sephadex column (Minkova et al., 2003) achieved a yield of 46\% with acceptable purity (established by Rito-Palomares et al., ${ }^{[5]}$ ).

Based on the information herein gathered, a protocol was proposed with a one-step extraction processin order to obtain both a good yield and a high grade of purity. This was accomplished by using an aqueous two-phase system with a posterior ultrafiltration, giving C-phycocyaninin a yield of 57\% and a purity of 3.9, thus surpassing the results of previous methodologies. It can be clearly seen that the preferred method should not be based on adsorption or elucidation, thus ruling out chromatography, because these processes diminish the yield of the extract (Cruz de Jesus, ${ }^{[74]}$ ).

\section{Conclusions}

To maximize the health benefits that may be obtained from nutraceuticals, such as C-phycocyanin, it is essential to seek innovative methods for their isolation and purification, and thus preserve the valuable properties of these bioactive substances from natural sources. The current review makes it evident that to obtain nutraceuticals from extracts and achieve good yield and high purity; it is convenient to use aqueous two-phase systems for extraction together with ultrafiltration for purification. It is also essential to consider the freshness and species of the primary biomass, as these factors heavily influence the concentration and viability of the desired phycobiliproteins and therefore affect the yield and purity.

\section{References}

1. Dillon, J.C., Phuc, A.P., Dubacq, J.P. Nutritional value of the alga Spirulina. (1995) World Rev Nutr Diet 77: 32-46.

2. Venkataraman, L.V. Spirulina platensis (Arthrospira): Physiology, Cell Biology and Biotechnologym, edited by Avigad Vonshak. (1997) J Applied Phycology 9(3): 295-296.

3. Belay, A. The potential application of Spirulina (Arthrospira) as a Nutritional and Therapeutic Supplement in Health Management. (2002) The J American Nutraceutical Association 5(2): 27-48.

4. Remirez, D., Ledón, N., González, R. Role of histamine in the inhibitory effects of phycocyanin in experimental models of allergic inflammatory response. (2002) Mediators Inflamm 11(2): 81-85.

5. Torres-Duran, P.V., Ferreira-Hermosillo, A., Juarez-Oropeza, M.A. Antihyperlipemic and antihypertensive effects of Spirulina maxima in an open sample of mexican population: a preliminary report. (2007) Lipids Health Dis 6: 33.

6. Lima, K., Facchinetti, A., Santos, C. Effects of different extracts of Spirulina on glycemic parameters in diabetic normal times. III Latin American Symposium of Food Science. (1999) Campinas.

7. Mittal, A., Suresh Kumar, P.V., Banerjee, S., Rao, A.R., et al. Modulatory potential of Spirulina fusiformis on carcinogen metabolizing enzymes in Swiss albino mice. (1999) Phytotherapy Research 13(2): 111-114.

8. Lee, J.B., Srisomporn, P., Hayashi, K., et al. Effects of structural modification of calcium spirulan, a sulfated polysaccharide from Spirulina platensis, on antiviral activity. (2001) Chem Pharm Bull (Tokyo) 49(1): 108-110.

9. Simsek, N., Karadeniz, A., Kalkan, Y., et al. Spirulina platensis feeding inhibited the anemia- and leucopenia-induced lead and cadmium in rats. (2009) J Hazard Mater 164(2-3): 1304-1309.

10. Karkos, P. D., Leong, S. C., Karkos, C. D., et al.. Spirulina in Clinical Practice: Evidence-Based Human Applications. (2011) Evid Based Complement Alternat Med 2011: 531053.

11. Gantt, E., Lipschultz, C. A., Grabowski, J., et al. Phycobilisomes from blue-green and red algae: isolation criteria and dissociation characteristics. (1979) Plant Physiology 63(4): 615-620.

12. Hoseini, S.M., Khosravi-Darani, K., et al. Nutritional and medical applications of Spirulina microalgae. (2013) Mini Rev Med Chem 13(8): 1231-1237.

13. Eriksen, N.T. Production of phycocyanin--a pigment with applications in biology, biotechnology, foods and medicine. (2008) Appl Microbiol Biotechnol 80(1): 1-14.

14. Khan, M., Varadharaj, S., Ganesan, L.P., et al. C-phycocyanin protects against ischemia-reperfusion injury of heart through involvement of p38 MAPK and ERK signaling. (2006) Am J Physiol Heart Circ Physiol 290(5): H2136-H2145.

15. Glazer, A.N., Lundell, D.J., Yamanaka, G., et al. The structure of a simple phycobilisome. (1983) Ann Microbiol (Paris) 134(1): 159-180.

16. Ocarra, P. Purification and N-terminal analyses of algal biliproteins. (1965) Biochem J 94(1): 171-174.

17. Bekasova, O. D., Muslimov, I. A., Krasnovskii, A. A. Fractionation of phycobilisomes from the blue-green alga Nostoc muscorum. (1984) Molekuliarnaia Biologiia 18(1): 262-271.

18. Swanson, R.V., Glazer, A.N. Separation of phycobiliprotein subunits by reverse-phase high-pressure liquid chromatography. (1990) Anal Biochem 188(2): 295-299.

19. Moreno, A., Bermejo, R., Talavera, E., et al. Purification, crystallization and preliminary X-ray diffraction studies of C-phycocyanin and allophycocyanin from Spirulina platensis. (1997) Acta Crystallogr D Biol Crystallogr 53(Pt 3): 321-326. 
20. Viskari, P.J., Colyer, C.L. Separation and quantitation of phycobiliproteins using phytic acid in capillary electrophoresis with laser-induced fluorescence detection. (2002) J Chromatogr A 972(2): 269-276.

21. Zolla, L., Bianchetti, M. High-performance liquid chromatography coupled on-line with electrospray ionization mass spectrometry for the simultaneous separation and identification of the Synechocystis PCC 6803 phycobilisome proteins. (2001) J Chromatogr A 912(2): $269-279$.

22. Bhaskar, S. U., Gopalaswamy, G., Raghu, R. A simple method for efficient extraction and purification of C-phycocyanin from Spirulina platensis Geitler. (2005) Indian J Exp Biol 43(3): 277-279.

23. Patel, A., Mishra, S., Pawar, R., et al. Purification and characterization of C-Phycocyanin from cyanobacterial species of marine and freshwater habitat. (2005) Protein Expr Purif 40(2): 248-255.

24. Niu, J.F., Wang, G.C., Lin, X.Z., et al. Large-scale recovery of C-phycocyanin from Spirulina platensis using expanded bed adsorption chromatography. (2007) J Chromatogr B Analyt Technol Biomed Life Sci 850(1-2): 267-276.

25. Soni, B., Trivedi, U., Madamwar, D. A novel method of single step hydrophobic interaction chromatography for the purification of phycocyanin from Phormidium fragile and its characterization for antioxidant property. (2008) Bioresour Technol 99(1): 188-194.

26. Yin, L., Xu, L., Yu, K., et al. Orthogonal test design for optimization of suitable conditions to separate C-phycocyanin from Spirulina platensis by high-speed counter-current chromatography using reverse micelle solvent system. (2011) J Sep Sci 34(11): 1253-1260.

27. Patil, G., Chethana, S., Sridevi, A.S., et al. Method to obtain C-phycocyanin of high purity. (2006) J Chromatogr A 1127(1-2): 76-81.

28. Fu, E., Friedman, L., Siegelman, H.W. Mass-spectral identification and purification of phycoerythrobilin and phycocyanobilin. (1979) Biochem J 179(1): 1-6.

29. Patil, G., Chethana, S., Madhusudhan, M.C., et al. Fractionation and purification of the phycobiliproteins from Spirulina platensis. (2008) Bioresour Technol 99(15): 7393-7396.

30. Rito-Palomares, M., Nuñez, L., Amador, D. Practical application of aqueous two-phase systems for the development of a prototype process for c-phycocyanin recovery from Spirulina maxima. (2001) Chem Tech Biotech 76(12): 1273-1280.

31. Seo, Y., Choi, W., Park, J., et al. Stable Isolation of Phycocyanin from Spirulina platensis Associated with High-Pressure Extraction Process. (2013) Int J Mol Sci 14(1): 1778-1787.

32. Moraes, C.C., Mazutti, M.A., Maugeri, F., et al. Modeling of ion exchange expanded-bed chromatography for the purification of C-phycocyanin. (2013) J Chromatogr A 1281: 73-78.

33. Ou, Y., Lin, L., Yang, X., et al. Antidiabetic potential of phycocyanin: Effects on KKAy mice. (2013) Pharm Biol 51(5): 539-544.

34. Cruz de Jesús, V. Optimizing a process for purifying C - phycocyanin from Spirulina maxima. Thesis, School of Higher Studies Cuautitlán Izcalli. (2005) Autonomous University of Mexico.

35. Benavides, J., Rito-Palomares, M. Potential aqueous two-phase processes for the primary recovery of colored protein from microbial origin. (2005) Engineering in Life Sciences 5(3): 259-266.

36. Silveira, S., Burkert, J.F., Costa, J.A., et al. Optimization of phycocyanin extraction from Spirulina platensis using factorial design. (2007) Bioresour Technol 98(8): 1629-1634.

37. Antelo, F. S., Anschau, A., Costa, J. A., Et al. (2010). Extraction and purification of C-phycocyanin from Spirulina platensis in conventional and integrated aqueous two-phase systems. J Braz Chem Soc 21(5): 921-926.

38. Maurya, S.S., Maurya, J.N., Pandey, V.D. Factors regulating phycobiliprotein production in cyanobacteria. (2014) Int J Curr Microbiol App Sci 3(5): 764-771.

39. Chaiklahan, R., Chirasuwan, N., Loha, V., et al. Separation and purification of phycocyanin from Spirulina sp. using a membrane process. (2011) Bioresour Technol 102(14): 7159-7164.

40. Cian, R.E., López-Posadas, R., Drago, S.R., et al. Immunomodulatory Properties of the Protein Fraction from Phorphyra columbina. (2012) J Agri Food Chem 60(33): 8146-8154.

41. Ducret, A., Sidler, W., Wehrli, E., et al. Isolation, characterization and electron microscopy analysis of a hemidiscoidal phycobilisome type from the cyanobacterium Anabaena sp. PCC 7120. (1996) Eur J Biochem 236(3): 1010-1024.

42. Yoshida, A., Takagaki, Y., Nishimune, T. Enzyme Immunoassay for Phycocyanin as the Main Component of Spirulina Color in Foods. (1996) Biosci Biotechnol Biochem 60(1): 57-60.

43. Bermejo-Bescós, P., Piñero-Estrada, E., Villar del Fresno, Á. M. Neuroprotection by Spirulina platensis protean extract and phycocyanin against iron-induced toxicity in SH-SY5Y neuroblastoma cells. (2008) Toxicol In Vitro 22(6): 1496-1502.

44. Glazer, A.N., Cohen-Bazire, G. Subunit structure of the phycobiliproteins of blue-green algae. (1971) Proc Natl Acad Sci U S A 68(7): 13981401.

45. He, J.A., Hu, Y.Z., Jiang, L.J. Photodynamic action of phycobiliproteins: in situ generation of reactive oxygen species. (1997) Biochimica et Biophysica Acta (BBA) Bioenergetics 1320(2): 165-174.

46. Li, B., Zhang, X., Gao, M., et al. Effects of CD59 on antitumoral activities of phycocyanin from Spirulina platensis. (2005) Biomed Pharmacother 59(10): 551-560.

47. MacColl, R., Habig, W., Berns, D. S. Characterization of Phycocyanin from Chroomonas Species. (1973) J Biol Chemistry 248(20): 70807086.

48. Piñero Estrada, J. E., Bermejo Bescós, P., Villar del Fresno, A. M. Antioxidant activity of different fractions of Spirulina platensis protean extract. (2001) Il Farmaco, 56(5-7): 497-500.

49. Kumar, D., Dhar, D., Pabbi, S., et al. Extraction and purification of C-phycocyanin from Spirulina platensis (CCC540). (2014) Indian J Plant Physiol 19: 184-188.

50. Chakdar, H., Saha, S., Pabbi, S. Chromatographic and spectroscopic characterization of phycocyanin and its subunits purified from Anabaena variabilis CCC421. (2014) Applied Biochemistry and Microbiology, 50(1), 62-68.

51. Moon, M., Mishra, S., Kim, C., et al. Isolation and characterization of thermostable phycocyanin from Galdieria sulphuraria. (2014) Korean Journal of Chemical Engineering 31(3): 490-495.

52. Abd El-Baky, H. H., El-Baroty, G. S. Characterization and bioactivity of phycocyanin isolated from Spirulina maxima grown under salt stress. (2012) Food \& Function 3(4): 381-388.

53. Gray, B. H., Lipschultz, C. A., Gantt, E. Phycobilisomes from a blue-green alga Nostoc species. (1973) J Bacteriology 116(1): $471-478$.

54. Madhyastha, H. K., Radha, K. S., Sugiki, M., et al. Purification of c-phycocyanin from Spirulina fusiformis and its effect on the induction of urokinase-type plasminogen activator from calf pulmonary endothelial cells. (2006) Phytomedicine 13(8): 564-569. 
55. Stec, B., Troxler, R. F., Teeter, M. M. Crystal structure of C-phycocyanin from Cyanidium caldarium provides a new perspective on phycobilisome assembly. (1999) Biophys J 76(6): 2912-2921.

56. Gantar, M., Simović, D., Djilas, S., et al. Isolation, characterization and antioxidative activity of C-phycocyanin from Limnothrix sp. strain 37-2-1. (2012) J Biotechnol 159(1-2): 21-26.

57. Liao, X., Zhang, B., Wang, X., et al. Purification of c-phycocyanin from Spirulina platensis by single-step ion-exchange chromatography. (2011) Chromatographia, 73(3): 291-296.

58. Abalde, J., Berancour, L., Torres, E., et al. Purification and characterization of phycocyanin from the marine cyanobacterium Synechococcus sp. 109201. (1998) Plant Science 136(1): 109-120.

59. ÓhEocha, C., Haxo, F. T. Some atypical algal chromoproteins. (1960) Biochimica et Biophysica Acta, 41(3), 516-520.

60. Kobayashi, Y., Siegelman, H. W., \& Hirs, C. H. W. C-phycocyanin from Phormidium luridum. Isolation of subunits. (1972). Arch Biochem Biophys 152(1): 187-198.

61. Torjesen, P. A., Sletten, K. C-phycocyanin from Oscillatoria agardhii: I. Some molecular properties. (1972) Biochimica et Biophysica Acta 263(2): 258-271.

62. Thangam, R., Suresh, V., Asenath Princy, W et al. C-Phycocyanin from Oscillatoria tenuis exhibited an antioxidant and in vitro antiproliferative activity through induction of apoptosis and G0/G1 cell cycle arrest. (2013). Food Chemistry, 140(1-2), 262-272.

63. Santiago-Santos, M. C., Ponce-Noyola, T., Olvera-Ramírez, R., et al. Extraction and purification of phycocyanin from Calothrix sp. (2004) Process Biochemistry, 39(12), 2047-2052.

64. Benedetti, S., Rinalducci, S., Benvenuti, F., et al. Purification and characterization of phycocyanin from the blue-green alga Aphanizomenon flos-aquae. (2006) J Chromatogr B Analyt Technol Biomed Life Sci. 833(1): 12-18.

65. Cai, C., Wu, L., Li, C., et al. Purification, crystallization and preliminary X-ray analysis of phycocyanin and phycoerythrin from Porphyra yezoensis Ueda. (2011). Acta Crystallographica Section F: Structural Biology and Crystallization Communications, 67(Pt 5), 579-583.

66. Ramos, A., Acién, F. G., Fernández-Sevilla, J. M., et al. Large-scale isolation and purification of C-phycocyanin from the cyanobacteria Anabaena marina using expanded bed adsorption chromatography. (2010) Journal of Chemical Technology \& Biotechnology 85(6): 783-792.

67. Huang, Z., Yang, F., Zheng, W. J., et al. Purification and Preparation of Active Subunits of C-phycocianin by a Protocol of Liquid-phase Isoelectric Focusing. (2006.) Chemical Journal of Chinese Universities 27(6): 1051-1054.

68. Moraes, C. C., da Costa Ores, J., Costa, J. A. V., et al. Recovery of C-phycocyanin in the presence of cells using expanded bed IEC. (2011) Chromatographia, 74(3): 307-312.

69. Ramos, A., Acién, F. G., Fernández-Sevilla, J. M., et al. Development of a process for large-scale purification of C-phycocyanin from Synechocystis aquatilis using expanded bed adsorption chromatography. (2011) J Chromatogr B 879(7-8): 511-519.

70. Bermejo, R., Ramos, A. Pilot scale recovery of phycocyanin from spirulina platensis using expanded bed adsorption chromatography. (2012) Chromatographia 75(5-6): 195-204.

71. Bermejo, R., Felipe, M. A., Talavera, E. M., et al. Expanded bed adsorption chromatography for recovery of phycocyanins from the microalga Spirulina Platensis. (2006) Chromatographia 63(1-2): 59-66.

72. Gupta, A., Sainis, J. Isolation of C-phycocyanin from Synechococcus sp., (Anacystis nidulans BD1). (2010) Journal of Applied Phycology, 22(3): 231-233.

73. Kao, O. H. W., Berns, D. S., Town, W. R. The characterization of C-phycocyanin from an extremely halo-tolerant blue-green alga, Coccochloris elabens. (1973) Bioche 131(1): 39-50.

74. Pleonsil, P., Soogarun, S., Suwanwong, Y. Anti-oxidant activity of holo- and apo-c-phycocyanin and their protective effects on human erythrocytes. (2013) Int J Bio Macromol 60: 393-398.

75. Song, W., Zhao, C., Wang, S. A Large-Scale Preparation Method of High Purity C-Phycocyanin. (2013) International journal of bioscience, biochemistry, bioinformatics. 3(4): 293.

76. Minkova, K., Gigova, L., Tchernov, A., et al. Isolation of Pure C-Phycocyanin from Arthrospira Maxima and Arthrospira Fusiformis by a Modified Non-Chromatographic Rivanol-Sulfate Procedure. (2007) Comptes rendus de l'Acad'emie bulgare des Sciences, 60(7): 797-800.

77. Minkova, K., Tchorbadjieva, M., Tchernov, A., et al. Improved procedure for separation and purification of Arthronema africanum phycobiliproteins. (2007) Biotechnol Lett 29(4): 647-651.

78. Chen, T., Wong, Y.S., Zheng, W. Purification and characterization of selenium-containing phycocyanin from selenium-enriched Spirulina platensis. (2006) Phytochemistry, 67(22): 2424-2430.

79. Yan, S.G., Zhu, L.P., Su, H.N., et al. Single-step chromatography for simultaneous purification of C-phycocyanin and allophycocyanin with high purity and recovery from Spirulina (Arthrospira) platensis. (2011) Journal of Applied Phycology, 23(1): 1-6.

80. Moraes, C. C., Kalil, S. J. Strategy for a protein purification design using C-phycocyanin extract. (2009). Bioresource Technol 100(21): 53125317.

81. Zhang, Y.M., Chen, F. A simple method for efficient separation and purification of c-phycocyanin and allophycocyanin from Spirulina platensis. (1999) Biotechnology Techniques 13(9): 601-603.

82. Sørensen, L., Hantke, A., Eriksen, N. T. Purification of the photosynthetic pigment C-phycocyanin from heterotrophic Galdieria sulphuraria. (2013) J Sci Food Agric 93(12): 2933-2938.

83. Marquardt, J., Senger, H., Miyashita, H., et al. Isolation and characterization of biliprotein aggregates from Acaryochloris marina, a Prochloronlike prokaryote containing mainly chlorophyll d. (1997) FEBS Lett 410(2-3): 428-432.

84. Sun, L., Wang, S., Qiao, Z. Chemical stabilization of the phycocyanin from cyanobacterium Spirulina platensis. (2006) Journal of Biotechnology 121(4): 563-569.

Online ISSN: 2377-0619

Journal Title: International Journal Food and Nutritional Science Journal Short Name: Int J Food Nutr Sci
Ommega Online Publishers

E-mail: foodscience@ommegaonline.org

Website: www.ommegaonline.org 